\title{
Synthesis of Tungsten Diselenide Nanoparticles by Chemical Vapor Condensation Method
}

\author{
Ekaterina VASILYEVA ${ }^{1 *}$, Albert G. NASIBULIN ${ }^{2,3}$, Maxim MAXIMOV ${ }^{1}$, \\ Oleg TOLOCHKO ${ }^{1}$, Anil SACHDEV ${ }^{4}$, Xingcheng XIAO ${ }^{4}$
}

\author{
${ }^{1}$ Saint Petersburg State Polytechnic University, 29 Polytechnicheskaya, Saint Petersburg, 195251 Russia \\ ${ }^{2}$ Aalto University School of Science, Puumiehenkuja 2, Espoo, Finland \\ ${ }^{3}$ Skolkovo Institute of Science and Technology, 100 Novaya st., Skolkovo, 143025, Russia \\ ${ }^{4}$ Material and Processes Lab, General Motors Research and Development Center, Warren, MI 48090- 9055, USA \\ crossref http://dx.doi.org/10.5755/j01.ms.21.3.7356
}

Received 16 June 2014; accepted 19 September 2014

\begin{abstract}
Crystalline tungsten diselenide ( $\mathrm{WSe}_{2}$ ) nanoparticles have been synthesized by a gas phase reaction using tungsten hexacarbonyl and elemental selenium as precursor. The WSe 2 nanoparticle morphology varies from the spherical shape to flake-like layered structures. Usually particles consist of 5-7 single layers of $\mathrm{WS}_{2}$. Mean size in smaller dimension are less than $5 \mathrm{~nm}$ and the number of layers decreased linearly with decreasing reaction time and concentration of carbonyl in the gas phase. The mean value of interlayer distance in $<0001>$ direction is comparable with the microscopic values. The selenium-to-tungsten atomic ratios in the interval of $2.07 \ldots 2.19$ were determined, approach to the stoichiometric ratio of 2:1. Main impurities are oxygen and carbon and strongly interrelated with carbonyl concentration in the gas phase.

Keywords: WSe2 nanoparticles; chemical vapor condensation; chemical synthesis; layered structures.
\end{abstract}

\section{INTRODUCTION}

Tungsten diselenide is a diamagnetic indirect semiconductor, which belongs to the family of group VIB layer-type transition metal dichalcogenides. It has a hexagonal structure consisting of single sandwiched Se and $\mathrm{W}$ layers. There is a strong covalent bonding within the Se$\mathrm{W}$-Se layers but weak van der Waals interactions between neighboring sandwich layers. The interlayer distance between $\mathrm{WSe}_{2}$ layers is about $6.5 \AA$. WSe 2 nanoparticles can exist in various allotropy forms such as polyhedrical, fullerene-like and nanotubular structures [1, 2]. Chalcogenides of $\mathrm{W}$ and Mo are well known as solid lubricant materials and widely used for creation of antifriction coating for loaded parts in such areas as aerospace, nuclear, cryogenics, machine tools, racing engines, cutting tools, die casting, small arms. As compare with other chalcogenides of transition metals forming layered structure like disulphides, diselenides, and ditellurides of molybdenum, titanium, vanadium, chromium and others suitable as lubricants, the tungsten chalcogenides have better oxidation resistance at high temperature, quite low friction coefficient and chemical stability as in high vacuum as well in atmospheric air conditions [3].

Generally the main characteristics of nanoparticles such as a size distribution, structure, morphology, and surface condition extremely depend on production technology $[4,5]$. As compared with other methods the chemical synthesis of nanoparticles in the gas phase is a rapidly growing field because of its versatile applicability

\footnotetext{
* Corresponding author. Tel.: +7-904-6011017; fax: +7-812-2973627. E-mail address: katrinfr@indox.ru (E. Vasilyeva)
}

to almost all materials and high rate of production capability with little agglomeration. Since the properties of these nanoparticles are basically determined by their mean size, size distribution, external shape, internal structure, and chemical composition, the characteristics of powders must be precisely controlled during the production of the nanoparticles [6]. The Chemical Vapor Condensation (CVC) process was used to produce pure iron, tungsten and tungsten disulphide nanoparticles in a variety of particles mean size less than $100 \mathrm{~nm}$ [6-8]. The structure of crystals has been found to depend on temperature, heating rate, reaction time, etc.

In the present work, we prepared $\mathrm{WSe}_{2}$ nanopowders by a thermal reaction between $\mathrm{W}(\mathrm{CO})_{6}$ and Se in a quartz tube under argon atmosphere by the CVC method. Elemental selenium powders were chosen in order to avoid the use of highly toxic $\mathrm{H}_{2} \mathrm{Se}$ gas. The effect of processing parameters on the microstructure and size of nanoparticles were investigated.

\section{EXPERIMENTAL}

Tungsten hexacarbonyl $\left(\mathrm{W}(\mathrm{CO})_{6}\right)$ and pure selenium supplied by Sigma-Aldrich were used as $\mathrm{W}$ and Se precursors, respectively. The basic setup for the CVC synthesis is similar to that described in literature elsewhere [7]. Carrier gas of high purity argon with permanent gas flow rate of $0.22 \mathrm{l} / \mathrm{min}$ was fed through a heated bubbling unit containing the metal organic precursor of tungsten hexocarbonyl $\left(\mathrm{W}(\mathrm{CO})_{6}\right)$ at the vaporization temperature. Vaporization temperature of tungsten carbonyl was optimized at $100^{\circ} \mathrm{C}$. The flow of carrier argon gas mixed with the precursor vapor passes through a heated pipe system into the heated tubular 
reactor. A specially designed two-zoned furnace allows fixing different temperatures in the reactor. The removable cartridge filled by crystalline selenium was located in the first, low-temperature zone of the reactor at the evaporation temperature of $600{ }^{\circ} \mathrm{C}$, where the selenium evaporated and mixed with carbonyl vapor. In the hightemperature zone, mixed gas was heated up to the carbonyl decomposing conditions and reaction of tungsten with selenium in the gas phase at the temperature of $800-1000^{\circ} \mathrm{C}$. All particles were well deposited on the internal surface of water-cooled chamber, from which powders can be scrapped off and collected.

Residence time of precursor into reaction zone and precursor concentration in gas phase were changed by varying of accelerating gas flow, which directly coming into the reaction zone.

All experiments were carried out with the excess of selenium in the gas phase in the second zone of reactor at the reaction condition and selenium nanoparticles always exist in final product. In order to remove the excess of selenium, as-prepared powder was heat-treated in the flow of hydrogen gas at the temperature of $300{ }^{\circ} \mathrm{C}$.

The DRON 2.0 diffractometer with monochromatic $\mathrm{Cu} \mathrm{K} \alpha$ radiation $(\lambda=0.154051 \mathrm{~nm})$ was used to determine the crystalline phase and structure analysis of the samples. The particle mean size was identified based on the width of diffraction peaks using the Scherer equation [9]. Chemical composition of produced nanoparticles was investigated by EDS method. The morphology and size distribution of nanoparticles were determined by means of high-resolution transmission electron microscopy (HRTEM) by using JEOL JEM-2200FS operated at $80 \mathrm{kV}$. Samples were prepared by dispersing in ethanol ultrasonically and dropped into the carbon-coated Cu-grids. Interlayer distance of $\mathrm{WSe}_{2}$ samples was evaluated as half of lattice parameter in $<0001>$ direction of hexagonal symmetry.

\section{RESULTS AND DISCUSSION}

Typical X-ray diffraction patterns for nanoparticles synthesized at the different accelerating gas flow rates are presented in Fig. 1. A set of well-defined diffractions peaks is seen, revealing pure $\mathrm{WSe}_{2}$ phase. As the accelerating gas flow rates increases, there is slight difference in the relative intensities of particular reflections and significant broadening of peaks in comparison with theoretical data for micron-sized powder. Estimation of average grain size with Sherrer's equation shows the mean grain size is estimated to be varies in range of $3-5 \mathrm{~nm}$ with increasing accelerating gas flow rate from 1.2 to $4.6 \mathrm{l} / \mathrm{min}$. X-ray diffraction pattern does not show any additional peaks except peaks $\mathrm{WSe}_{2}$, just trases of unreacted crystalline selenium (at $2 \Theta$ of $23-27 \mathrm{deg}$ ) was observed at the highest flow rate of $4.6 \mathrm{l} / \mathrm{min}$.

The typical appearance of synthesized powders is depicted in Fig. 2. Some agglomeration of nanoparticles can be observed and the powders provide nested onion structure and flat graphene-like structure. In our experiments graphene-like structure was observed first time as compare with tungsten disulphide nanoparticles with only closed fullerene-like structure produced by the same technique [7]. Graphene is a single layer of carbon atoms densely packed into a benzene-ring structure, and is widely used to describe many structures including graphite, large fullerenes, nanotubes, etc. Planar graphene itself has been presumed not to exist in a free state, being unstable with respect to forming curved structures such as fullerenes and nanotubes.

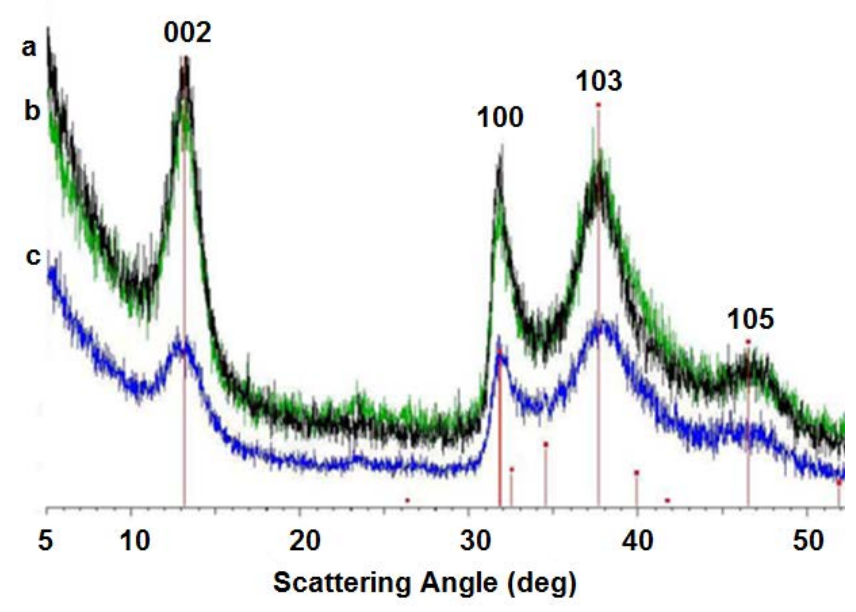

Fig. 1. XRD patterns of $\mathrm{WSe}_{2}$ nanoparticles synthesized under different conditions: reaction zone temperature $900{ }^{\circ} \mathrm{C}$, carrier gas flow rate $1 \mathrm{l} / \mathrm{min}$, accelerating gas (a) $2.4 \mathrm{l} / \mathrm{min}$, (b) $4.6 \mathrm{l} / \mathrm{min}$, (c); carrier 0.35 and accelerating gas $1.2 \mathrm{l} / \mathrm{min}$.
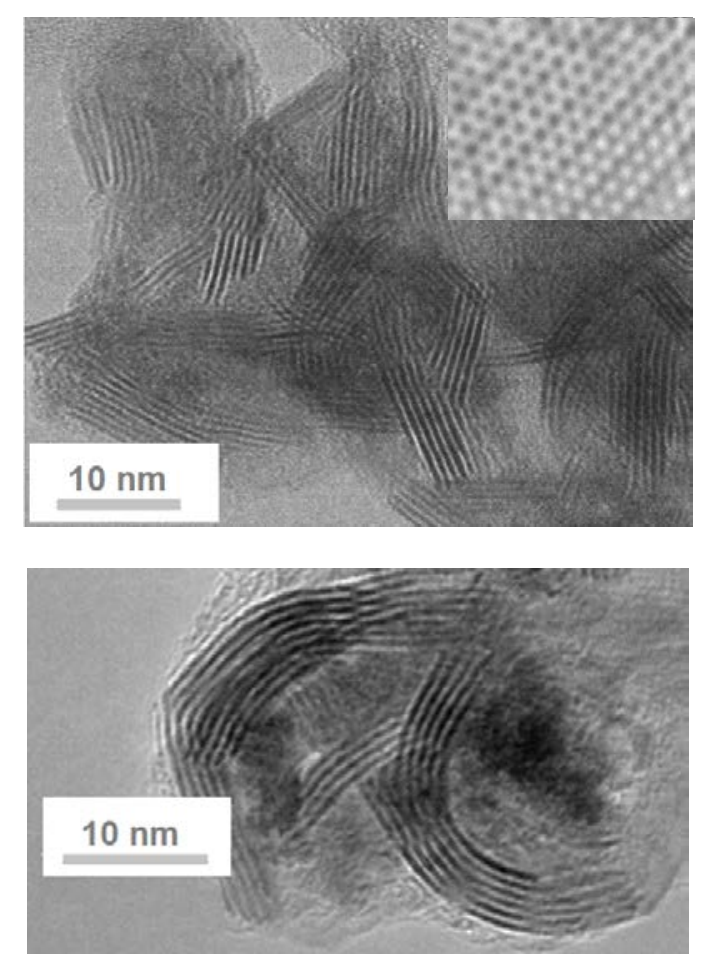

Fig. 2. TEM microphotographs of $\mathrm{WSe}_{2}$ nanoparticles. Insertion shows hexagonal graphene-like structure of layers

Synthesized graphene-like structures may contain foreign atoms intercalated between the Se-W-Se layers, which cause weakening of the bonds and modification of the crystalline structure. The presence of such atoms creating a sub-structure with weak long-range order may be supported by broadened diffraction. Thus, presence of foreign atoms with the different sizes of graphene-like particles leads to appearance defects such as dislocations in 
Table 1. Chemical composition of samples produced at different accelerating gas flow rates

\begin{tabular}{|c|c|c|c|}
\hline Concentration $\mathrm{W}(\mathrm{CO})_{6}$ in gas phase/residence time & $0.2 \mathrm{~mol} \%$ /0.35 sec & $0.36 \mathrm{~mol} \%$ /0.66 sec & $2.28 \mathrm{~mol} \%$ /4.28 sec \\
\hline C K & 0.45 & 1.9 & 17.53 \\
\hline O K & 2.94 & 6.59 & 26.25 \\
\hline Se K & 65.15 & 62.87 & 38.58 \\
\hline W L & 31.46 & 28.64 & 17.64 \\
\hline Total & 100 & 100 & 100 \\
\hline
\end{tabular}

structure and increasing of interlayer distance and leads to the curvature layered sheets. As it is known from properties of graphene, appearing in hexagonal structure number of pentagonal lattice cells leads to formation balllike shape and fullerene-like structure, in case of heptagon cells bias spontaneous curvature of sheets goes in longitudinal direction, forming different kinds of nanoparticles morphology [10].

Due to laminar gas flow in the vertical-type reactor, the residence time between tungsten and selenium decreases almost linearly with increasing gas flowing rate. The presence of location errors and non-periodicity of the structures causes the widening of diffraction peaks, which may decrease the apparent particle's mean size. In layered structures nonperiodicity and lattice defects appear due to intercalation with the foreign atoms and molecules in interlayer spacing.

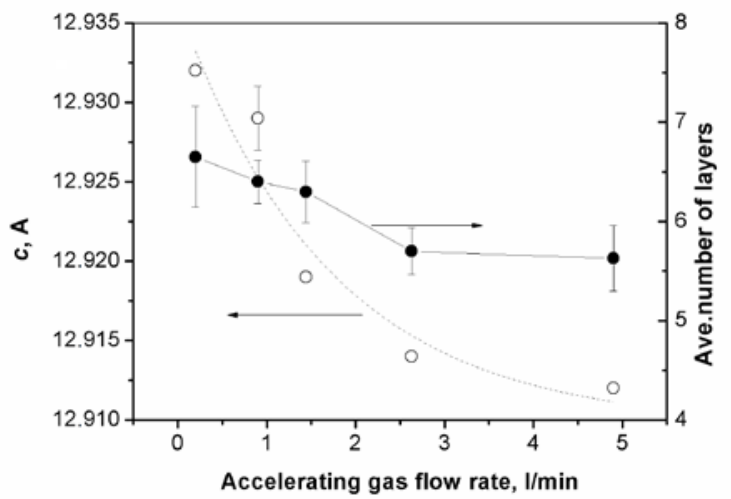

Fig. 3. Dependence of lattice parameter in $\mathrm{z}$ direction (c) and average layers number per particle evaluated from TEM on accelerating gas flow rate (different carbonyl concentration in the gas phase)

Chemical compositions of powders are presented in Table 1. The selenium-to-tungsten atomic ratios of 2.07, 2.19 and 2.19 were determined respectively, more than the stoichiometric ratio of $2: 1$. It is suggested that non-reacted raw materials may remain in powders. As we can see from the table, content of impurities $\mathrm{C}$ and $\mathrm{O}$ increased with decreasing accelerated gas flow rate. It is in correspondence with increasing of carbonyl concentration in gas phase. It means that main impurities come from the carbonyl as product of its decomposition, but not from the purified argon gas. Nanoparticles structure can be intercalated mostly CO molecules, because its dissociation is not intensively going in applied temperature interval.Results of lattice parameter $c$ (in $z$ direction) under different accelerating gas flow rates are presented in Fig. 3.

Experimental data shows increased lattice parameter and interlayer distance with decreasing accelerating gas flow rate, it should be compared with value of spacing in $<0001>$ direction for compact $2 \mathrm{H}-\mathrm{WSe}_{2}$ in equilibrium state $\mathrm{c}=12.98 \AA$ [11]. The decreasing of measured values of lattice parameters as compare with bulk one may be explained by the compressive stresses, which is common for nanoparticles. Interlayer distance between triple hexagonal planes in such structures can be evaluated like $c / 2$.

Experimentally it was found out that number of layers in nanoparticles structure decrease with increasing of accelerating gas flow rate leading to decreasing of tungsten hexacarbonyl concentration in the gas phase (Fig. 3) and residence time of pyrolysis products in the gas phase.

Thus, in correspondence with the measured interlayer distance of produced $\mathrm{WSe}_{2}$ samples, intercalation of nanoparticles structure by foreign atoms comes from carbonyl. Decreasing gas flow rate leads to increasing concentration of carbonyl in gas phase and increasing interlayer distance of the nanoparticles. The carbonyl pyrolysis reaction and monoxide non-intensive decomposition happens at the same time in used temperatures interval. Thus there is high possibility that structures contain a number of monoxide atoms. Nanoparticles with lower interlayer spacing mostly form flat structures like graphite and content more hexagonal layers in structure with reduced number of defects. So the changing accelerating gas flow rate makes it possible to regulate nanoparticles morphology.

\section{CONCLUSIONS}

This study has demonstrated the possibility to produce $\mathrm{WSe}_{2}$ nanoparticles by a chemical vapor condensation method. The powder particles have uniform size distribution with a mean size less than $5 \mathrm{~nm}$ in <0001> direction and decreases linearly with decreasing precursor concentration in gas phase and residence time. Nanoparticles have flat graphene-like structure and different type of curviness. In most of samples the interplanar distance in the $\left\{\begin{array}{lll}0 & 0 & 01\end{array}\right\}$ direction is comparable with etalon for micron powders. Increasing of carrier gas flow and residence time leads to formation number of lattice defects due to intercalation and expansion of interlayer distance. Main impurities are oxygen and carbon and their quantity increases with increasing carbonyl concentration. Synthesized $\mathrm{WSe}_{2}$ nanopowder has 
potential application as solid lubricants and as additives in the lubrication oils.

\section{Acknowledgments}

We gratefully acknowledge to Dr. Eric W. Shneider for support and Misle Tessema from Chemical \& Analysis Group, GM R\&D Center for support with some of X-Ray diffraction measurements and calculation. We are indebted to Dr. I. Anoshkin for assistance with some TEM images and EDS analysis of nanoparticles samples.

This work was supported by Ministry of Education and Science of Russian Federation (Project DOI: RFMEFI58114X0006).

\section{REFERENCES}

1. Tenne, R. Advances in the Synthesis of Inorganic Nanotubes and Fullerene-like Nanoparticles Angewandte Chemic International Edition 42 (51) 2003: pp. 24-32.

2. Deepak, F. L., Tenne, R. Gas-phase Synthesis of Inorganic Fullerene-like Structures and Inorganic Nanotubes Central European Journal of Chemistry 6 (3) 2008: pp 373-389.

3. Lansdown, A. R. Molybdenium Disulphide Lubrication, Elsevier, Swansea, 1999.

4. Nasibulin, A. G., $\quad$ Moisala, A., $\quad$ Brown, D. P., Kauppinen, E. I. Carbon Nanotubes and Onions from Carbon Monoxide using $\mathrm{Ni}(\mathrm{acac})_{2}$ and $\mathrm{Cu}(\mathrm{acac})_{2}$ as Catalyst Precursors Carbon 41 (14) 2003: pp. 2711-2724.
5. Sugimoto, T. Fine Particles-Synthesis, Characterization and Mechanism of Growth, Marcel Dekker, New York, 1996.

6. Choi, C. J., Kim, B. K., Tolochko, O., Li-Da. Preparation and Characterization of Magnetic $\mathrm{Fe}, \mathrm{Fe} / \mathrm{C}$ and $\mathrm{Fe} / \mathrm{N}$ Nanoparticles Synthesized by Chemical Vapor Condensation Process Reviews on Advanced Materials Science 5 (5) 2003: pp. 487-492.

7. Vasilyeva, E. S., Tolochko, O. V., Kim, B. K., Lee, D. W., Kim, D. S. Synthesis of Tungsten Disulphide Nanoparticles by the Chemical Vapor Condensation Method Microelectronics Journal 40 (4-5) 2009: pp. 687-691.

8. Kim, D., Vasilieva, E. S., Nasibulin, A. G., Lee, D. W., Tolochko, O. V., Kim, B. K. Aerosol Synthesis and Growth Mechanism of Magnetic Iron Nanoparticles Materials Science Forum 534-536 (1) 2007: pp. 9-12.

9. Jenkins, R., Snyder, R. L. Introduction to X-ray Powder Diffractometry. John Wiley \& Sons Inc., 1996: pp.89-91. http://dx.doi.org/10.1002/9781118520994

10. Chuvilin, A., Kaiser, U., Bichoutskaia, E., Besley, N. A., Khlobystov, A. N. Direct Transformation of Graphene to Fullerene Nature Chem 450 (2) 2010: pp.450-453.

11. Kear, B. H., Strutt, P. R. Chemical Processing and Applications for Nanostructured Materials Nanostructured Materials $6(1-4)$ 1995: pp. $227-236$. 